\title{
Associação entre Prevalência de Inatividade Física e Indicadores de Condição Socioeconômica em Adolescentes
}

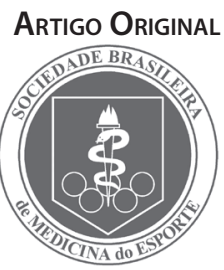

\section{Association Between Prevalence of Physical Inactivity and Indicators of Socio-Economic Status in Adolescents}

\author{
José Cazuza de Farias Júnior 1,2,3 \\ 1. UFPB/CCS/DEF - João Pessoa, PB, \\ Brasil \\ 2. UFSC/CDS/PPGEF - Florianópolis, \\ SC, Brasil \\ 3. Bolsista Capes
}

\section{Endereço para correspondência:} Rua: Capitão Romualdo de Barros, 997 - Apto. 303 - Bl. B1, Carvoeira Florianópolis, SC - CEP: 80040-600 Fone: (48) 3209-7713

Email: jcazuzajr@hotmail.com

Submetido em 24/04/2007 Versão final recebida em 23/07/2007 Aceito em 23/10/2007

\begin{abstract}
RESUMO
Objetivo: A redução nos níveis de atividade física na população jovem tem sido amplamente descrita em vários países, sobretudo em alguns subgrupos dessa população. O objetivo deste estudo foi determinar a prevalência de inatividade física em adolescentes escolares do ensino médio do município de João Pessoa - PB, e analisar sua associação com indicadores de condição socioeconômica. Metodologia: Participaram do estudo 2.566 adolescentes (1.132 rapazes e 1.434 moças), de 14 a 18 anos de idade $(16,5 \pm 1,17)$. Foram levantadas informações demográficas (sexo e idade), socioeconômicas (trabalho, tipo de escola, classe econômica, escolaridade dos pais), e mediu-se o nível de atividade física (kcal/kg/dia), mediante utilização de um diário de atividade física. Foram classificados como fisicamente inativos os adolescentes com demanda energética diária $<37 \mathrm{kcal} / \mathrm{kg} /$ dia. Na análise multivariável recorreu-se à regressão de Poisson, tendo a razão de prevalência como medida de associação. Resultados: Cerca de seis em cada dez adolescentes foram classificados como fisicamente inativos $(55,9 \%, n=1.435)$, com prevalência estatisticamente mais elevada nas moças $(64,2 \%)$ do que nos rapazes (45,5\%; $p<0,001)$. A prevalência de inatividade física se associou positivamente com a condição socioeconômica, indicando maior prevalência de inatividade física nos adolescentes que não trabalhavam (rapazes $R P=2,22 ;$ IC95\%=1,62-3,04 e moças $R P=1,52 ;$ IC95\%=1,19-1,93), e nas moças cujos pais apresentavam maior nível de escolaridade $(\mathrm{RP}=1,20 ; \mathrm{IC} 95 \%=1,05-1,35)$, comparados, respectivamente, aos que trabalham e cujos pais tinham menor nível de escolaridade. Conclusão: A prevalência de inatividade física foi alta, principalmente nas moças. Adolescentes que pertenciam aos estratos socioeconômicos mais privilegiados se mostraram mais expostos à inatividade física, sobretudo as moças.
\end{abstract}

Palavras-chave: adolescente, atividade física, inatividade física, condição socioeconômica.

\begin{abstract}
Objective: The decrease in the levels of physical activity in the young population has been described thoroughly in several countries, above all in some subgroups of that population. The objective of this study was to determine the prevalence of physical inactivity in high school adolescents from the João Pessoa city - PB and to analyze its association with indicators of socio-economic status. Methodology: 2,566 adolescents (1,132 boys and 1,434 girls), ages between 14-18 years (16.5 \pm 1.17$)$ participated in this study. Demographic (sex and age) and socio-economic data (work, school type, economic class, and parents' educational background) were raised. Besides that, the level of physical activity ( $\mathrm{kcal} / \mathrm{kg} /$ day) was measured through a diary of physical activity. The adolescents were classified as physically inactive when had daily energy demand $<37 \mathrm{kcal} / \mathrm{kg} / \mathrm{day}$. The ratio prevalence (RP), with respective reliability intervals of 95\% (RI95\%), was used as association measure. Results: The prevalence of physical inactivity positively associated with the socio-economic status, showing higher prevalence in the adolescents who did not work (boys $\mathrm{RP}=2.22 ; \mathrm{R} \mid 95 \%=1.62-3.04$ and girls $\mathrm{RP}=1.52 ; \mathrm{R} \mid 95 \%=1.19$ 1.93), and in the girls whose parents presented higher educational background (girls $R P=1.20 ; R \mid 95 \%=1.05-1.35$ ), compared with the adolescents who worked and the ones whose parents had lower educational background, respectively. Conclusion: The prevalence of physical inactivity was high, mainly in the girls. Adolescents who belonged to more priviledged socio-economic strata were more exposed to physical inactivity.
\end{abstract}

Keywords: adolescent, physical activity, physical inactivity, socio-economic status.

\section{INTRODUÇÃO}

Apesar da reconhecida importância da atividade física para a saúde e o bem-estar, uma grande proporção de adolescentes não consegue alcançar níveis satisfatórios de atividade física. Estudos internacionais ${ }^{(1-4)}$ e nacionais ${ }^{(5-7)}$ têm demonstrado prevalência elevada de inatividade física na população jovem, assim como, uma tendência de declínio no nível de prática de atividade física nas últimas décadas $(8,9)$.

Níveis insuficientes de atividade física durante a infância e ado- lescência têm sido freqüentemente associados ao acúmulo excessivo de gordura corporal, alterações desfavoráveis no perfil dos lipídios sanguíneos, níveis elevados de pressão arteria|(10-12), exposição a outros comportamentos de risco à saúde ${ }^{(13)}$, e maior probabilidade de inatividade física na fase adulta da vida ${ }^{(14,15)}$.

Aumentar os níveis de prática de atividade física na população jovem, tem representado uma das principais prioridades de saúde pública, em países de diferentes níveis de desenvolvimento socioeco- 
nômico. Entretanto, apesar dos esforços demandados, os programas de promoção da atividade física têm-se mostrado pouco efetivos em mudar os níveis de atividade física dos adolescentes ${ }^{(16-19)}$. Tem sido amplamente descrito que a melhoria na efetividade desses programas está condicionada, entre outros fatores, a um melhor conhecimento sobre os fatores potenciais que influenciam os hábitos de atividade física dos jovens.

Variáveis de diferentes dimensões (intra-pessoal, psicológica, interpessoal, ambiental) se associam aos níveis de prática de atividade física em adolescentes ${ }^{(20-22)}$. Entre as variáveis intra-pessoais, com exceção do sexo e idade, a condição socioeconômica tem sido pouco investigada, sobretudo em adolescentes brasileiros. Os estudos realizados e que puderam ser localizados, foram desenvolvidos com adolescentes da Região Sul ${ }^{(5,7,23)}$ e Sudeste do Brasil|(24), não sendo localizado nenhum estudo com adolescentes da região nordeste.

A análise da relação entre o nível de atividade física e a condição socioeconômica dos adolescentes tem produzido resultados conflitantes. Enquanto alguns estudos demonstram relação positiva ${ }^{(6,22,25-28)}$, outros apresentam uma relação inversa ${ }^{(5,23,29)}$ e, por vezes, nenhuma $\operatorname{associação(~}^{(7)}$.

A distribuição da prevalência de adolescentes fisicamente ativos/ inativos, em função de indicadores demográficos e socioeconômicos, e a magnitude da associação entre fatores de influência e o nível de atividade física, representam informações importantes para nortear o planejamento, desenvolvimento e avaliação dos programas de promoção da atividade física.

Investigar a relação entre a condição socioeconômica e o nível de atividade física, considerando diferentes contextos de prática, poderá suscitar hipóteses que conduzam a novas investigações que ajudem a esclarecer quais os fatores que levam a diferenças no tipo e grau de envolvimento com atividade física, em adolescentes de diferentes condições socioeconômicas. Nesse sentido, procurou-se determinar a prevalência de inatividade física em adolescentes escolares do ensino médio do município de João Pessoa - PB, e analisar sua associação com indicadores de condição socioeconômica.

\section{Metodologia}

O estudo sobre prevalência de inatividade física e associação com indicadores de condição socioeconômica foi desenvolvido a partir de um estudo epidemiológico transversal "Comportamentos de risco à saúde em adolescentes escolares do ensino médio do município de João Pessoa - PB", realizado em 2005. Os protocolos de intervenção no estudo foram aprovados pelo Comitê de Ética em Pesquisa da Universidade Federal da Paraíba.

Na estimativa do tamanho da amostra, considerou-se uma prevalência de inatividade física de 60\%, intervalo de confiança 95\% (IC95\%), erro estimado de 3 pontos percentuais e um efeito de delineamento 2 (efeito do design amostral - deff). Dados esses parâmetros e considerando uma população de 31.125 escolares do ensino médio (ano-base 2005), estimou-se uma amostra de 1.984. A esse valor foi acrescido 20\% para possíveis perdas, totalizando uma amostra de 2.380. Como este estudo era parte de uma investigação ampla, aplicou-se o questionário em 2.996 escolares do ensino médio.

O processo de amostragem foi realizado em dois estágios a partir dos 65 estabelecimentos de ensino médio (públicos e privados), existentes no município de João Pessoa - PB. No estágio inicial, foram selecionadas de forma aleatória e proporcional (tamanho e tipo de escola), 16 escolas, sendo 10 estaduais e 6 privadas.

No segundo estágio, selecionou-se em cada escola sorteada para participar do estudo, após solicitação prévia do termo de anuência dos gestores das escolas, as turmas para compor a amostra. De forma aleatória e proporcional (tipo de escola e série do ensino médio), foram selecionadas 110 turmas, das 100 previstas $(2.996 \div 30=100$ ), prevendo-se que algumas turmas poderiam ter número inferior a 30 alunos. Todos os escolares da turma sorteada, que estavam em sala de aula no dia da coleta, foram considerados elegíveis para participar do estudo. $O$ termo de consentimento negativo foi utilizado para obter a anuência pais e/ou responsáveis.

Foram excluídos os escolares com idade inferior a 14 anos $(n=77)$ e superior a 18 anos de idade ( $n=134)$, e os que preencheram de forma incompleta as informações sobre as atividades físicas praticadas ( $\mathrm{n}=219$; 107 rapazes e 112 moças). Desse modo, foram analisados dados completos sobre atividade física em 2.566 escolares (1.132 rapazes e 1.434 moças), de 14 a 18 anos de idade $(16,5 \pm 1,17)$.

Na coleta de dados, uma equipe composta por quatro estagiários, previamente treinados em dois estudos piloto, aplicou um questionário ${ }^{(30)}$ em sala de aula, que requeria 25 a 30 minutos para seu preenchimento pelos escolares. Os escolares foram questionados sobre as seguintes informações: i) demográficas (sexo e idade); ii) socioeconômicas (trabalho, tipo de escola, classe econômica, escolaridade dos pais) e; iii) atividades físicas praticadas.

A idade dos adolescentes foi determinada, com base na diferença entre a data de nascimento e a data da coleta de dados, sendo adotado o seguinte procedimento: 14 a 14,99 como 14 anos, e assim sucessivamente até os 18 anos de idade.

Foi considerado como trabalhador o escolar que exercia qualquer tipo de trabalho, desde que recebesse remuneração, com carteira assinada ou não. O tipo de escola foi definido previamente no instrumento, conforme registro da escola na Secretaria de Educação do Estado da Paraíba (escolas públicas estaduais e escolas privadas).

Para a definição da classe econômica, recorreu-se às diretrizes propostas pela Associação Brasileira de Empresas de Pesquisa (ABEP) (31), que agrupa as pessoas nas classes A (alta), B, C, D e E (baixa), a partir da acumulação de bens materiais, das condições de moradia, número de empregados domésticos e do nível de escolaridade do chefe da família. Por apresentarem uma pequena quantidade de sujeitos, as categorias $A$ e $E$ foram reagrupadas em $A, B$ e $D, E$, permanecendo a $C$ isoladamente.

A escolaridade dos pais dos adolescentes foi considerada segundo o número de anos completos de escolaridade do chefe da família (pai/mãe), sendo adotadas as seguintes categorias: $\leq 4$ anos, 5-8 anos, 9-11 anos e $\geq 12$ anos.

O nível de atividade física foi determinado mediante estimativa da demanda energética diária (kcal/kg/dia) (32), a partir das atividades registradas pelos escolares (36 períodos de 30 minutos), em um diário de atividade física(30), durante três dias da semana (6 às 24 horas/dia), sendo um dia do final de semana e dois dias do meio da semana. Foi considerado como fisicamente inativo, o adolescente que apresentou uma média ponderada da demanda energética diária dos três analisados $<37 \mathrm{kcal} / \mathrm{kg} / \mathrm{dia}^{(33)}$.

Para avaliar a associação bruta entre variáveis indicativas das condições socioeconômicas (trabalho, tipo de escola, classe econômica, escolaridade dos pais) e a inatividade física, utilizou-se à medida de razão de prevalência (RP), com respectivos intervalos de confiança de 95\% (IC 95\%). O teste do qui-quadrado foi empregado para avaliar heterogeneidade ou tendência linear. Na análise multivariável, utilizou-se a regressão de Poisson para estimar a associação independente 
da inatividade física (fisicamente ativo=0 e fisicamente inativo=1) com as variáveis indicativas de condição socioeconômica. Ambas as análises foram realizadas com estratificação da amostra por sexo, tendo em vista que os fatores que podem influenciar a atividade física diferem entre rapazes e moças. Todos os procedimentos de análise estatística foram realizados no programa Stata 9.0, com nível de significância de 5\%.

\section{RESULTADOS}

Como principais características demográficas e socioeconômicas da amostra estudada, destacam-se: adolescentes do sexo feminino (55,8\%), não trabalhavam (89,12\%), pertenciam às classes econômicas de nível alto $(A, B=31,6 \%)$ e médio-baixo ( $C=40,4 \%)$, e pais com nove ou mais anos de escolaridade (60,8\%) - tabela 1.

Aproximadamente seis em cada dez (55,9\%, n=1.435) escolares do ensino médio do município de João Pessoa - PB, foram classificados como fisicamente inativos (<37 kcal/kg/dia). A figura 1 mostra a prevalência de inatividade física conforme o sexo e a idade dos escolares.

Tabela 1. Características demográficas e socioeconômicas dos adolescentes escolares do ensino médio, João Pessoa - PB, 2005.

\begin{tabular}{|c|c|c|}
\hline Variável & $\mathrm{n}$ & $\%$ \\
\hline \multicolumn{3}{|l|}{ Sexo } \\
\hline Masculino & 1.132 & 44,2 \\
\hline Feminino & 1.434 & 55,8 \\
\hline \multicolumn{3}{|c|}{ Idade (anos) } \\
\hline 14 & 297 & 11,6 \\
\hline 15 & 622 & 24,3 \\
\hline 16 & 624 & 24,3 \\
\hline 17 & 637 & 24,8 \\
\hline 18 & 386 & 15,0 \\
\hline \multicolumn{3}{|l|}{ Trabalho } \\
\hline Sim & 279 & 10,9 \\
\hline Não & 2.287 & 89,1 \\
\hline \multicolumn{3}{|c|}{ Tipo de escola } \\
\hline Pública & 1.749 & 68,2 \\
\hline Privada & 817 & 31,8 \\
\hline \multicolumn{3}{|c|}{ Classe econômica } \\
\hline$A, B$ & 723 & 31,6 \\
\hline C & 926 & 40,4 \\
\hline$D, E$ & 642 & 28,0 \\
\hline \multicolumn{3}{|c|}{ Escolaridade dos pais* } \\
\hline$\leq 4$ anos & 431 & 16,1 \\
\hline 5-8 anos & 595 & 23,1 \\
\hline 9-11 anos & 796 & 31,1 \\
\hline$\geq 12$ anos & 744 & 29,7 \\
\hline
\end{tabular}

*Chefe da família

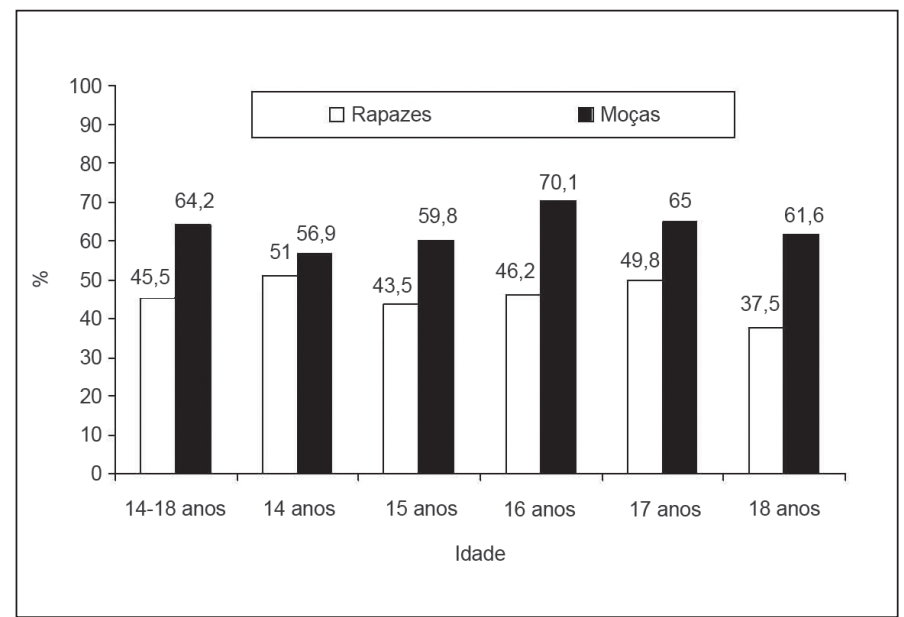

Figura 1. Prevalência de inatividade física conforme sexo e idade dos adolescentes escolares do ensino médio, João Pessoa - PB, 2005.

A prevalência de inatividade física foi mais elevada nas moças (64,2\%) do que nos rapazes $(45,5 \%, p<0,001 ; R P=1,41$; IC $95 \%=1,30-1,51)$, mantendo-se as diferenças em todas as idades estudadas. A prevalência de inatividade física não apresentou tendência de crescimento linear com o avanço da idade, tanto nos rapazes $(p=0,40)$ quanto nas moças $(p=0,26)$.

Na tabela 2, estão descritas as prevalências e razões de prevalência de inatividade física conforme indicadores de condição socioeconômica. A prevalência de inatividade física foi estatisticamente mais elevada nos adolescentes que não trabalhavam (rapazes e moças), nos rapazes que estudavam em escolas privadas, e nas moças que pertenciam às classes econômicas mais privilegiadas $(A, B)$ e cujos pais tinham maior grau de escolaridade.

$\mathrm{Na}$ análise bruta (tabela 2), a inatividade física se associou com o trabalho, tipo de escola, classe econômica e o grau de escolaridade dos pais. A chance de ser fisicamente inativo foi aproximadamente duas a três vezes maior nos adolescentes que não trabalhavam (rapazes $\mathrm{RP}=2,22$ e moças $\mathrm{RP}=1,52$ ), 26\% maior nos rapazes que estudavam em escolas privadas ( $\mathrm{RP}=1,26)$, e 13\% a 20\% maior nas moças que pertenciam às classes econômicas mais privilegiadas (classe econômica $A, B$ : $R P=1,13$ ) e eram filhas de pais com doze ou mais anos de escolaridade (escolaridade dos pais $\geq 12$ anos: $R P=1,20$ ), respectivamente.

Mesmo depois de ajustado para demais variáveis do modelo e idade dos escolares - análise multivariável (tabela 3), trabalho, nos adolescentes de ambos os sexos, e o grau de escolaridade dos pais, nas moças, continuaram associados positivamente com a inatividade física, indicando maior probabilidade de inatividade física nos adolescentes que não trabalhavam ( $R P=2,18$ nos rapazes e $R P=1,47$ nas moças) e nas moças cujos pais tinham maior grau de escolaridade ( $R P=1,18$ escolaridade 9-11 anos e $\mathrm{RP}=1,23$ escolaridade $\geq 12$ anos), tendo como referência os que não trabalhavam e cujos pais tinham baixo grau de escolaridade ( $\leq 4$ anos).

O tipo de escola, nos rapazes, e a classe econômica nas moças, que se mostraram estatisticamente associados à inatividade física na análise bruta, perderam significância estatística na análise multivariável, enquanto que a classe econômica e o grau de escolaridade dos pais, nos rapazes, e o tipo de escola, nas moças, continuaram dissociados da inatividade física. 
Tabela 2. Prevalências e razões de prevalência (RP) de inatividade física conforme indicadores da condição socioeconômica, João Pessoa - PB, 2005.

\begin{tabular}{|c|c|c|c|c|c|c|}
\hline \multirow{2}{*}{ Variável } & \multicolumn{3}{|c|}{ Rapazes } & \multicolumn{3}{|c|}{ Moças } \\
\hline & $\mathrm{n}$ & $\%$ & RP (IC95\%) & $\mathrm{n}$ & $\%$ & RP (IC95\%) \\
\hline Trabalho & & $p<0,001$ & & & $p<0,001$ & \\
\hline Sim & 32 & 21,9 & 1 & 39 & 43,3 & 1 \\
\hline Não & 472 & 48,8 & $2,22(1,62-3,04)$ & 862 & 65,9 & $1,52(1,19-1,93)$ \\
\hline Tipo de escola & & $p<0,001$ & & & $p=0,07$ & \\
\hline Pública & 314 & 41,8 & 1 & 683 & 62,8 & 1 \\
\hline Privada & 201 & 52,9 & $1,26(1,11-1,43)$ & 237 & 68,3 & $1,08(0,99-1,18)$ \\
\hline Classe econômica & & $p=0,54^{*}$ & & & $p=0,02^{*}$ & \\
\hline$A, B$ & 174 & 49,0 & $1,02(0,86-1,21)$ & 217 & 68,0 & $1,13(1,01-1,27)$ \\
\hline C & 142 & 39,6 & $0,82(0,68-1,01)$ & 325 & 65,4 & $1,08(0,90-1,21)$ \\
\hline$D, E$ & 113 & 47,9 & 1 & 219 & 60,0 & 1 \\
\hline Escolaridade dos pais ${ }^{\dagger}$ & & $p=0,14^{*}$ & & & $p<0,001^{*}$ & \\
\hline$\leq 4$ anos & 56 & 44,8 & 1 & 135 & 58,4 & 1 \\
\hline $5-8$ anos & 98 & 41,5 & $0,92(0,66-1,28)$ & 180 & 58,1 & $0,99(0,86-1,14)$ \\
\hline 9-11 anos & 160 & 45,3 & $1,01(0,74-1,37)$ & 314 & 66,4 & $1,13(1,01-1,28)$ \\
\hline$\geq 12$ anos & 193 & 48,7 & $1,08(0,80-1,46)$ & 274 & 69,9 & $1,20(1,05-1,35)$ \\
\hline
\end{tabular}

*Teste para tendência linear

${ }^{\dagger}$ Chefe da família

Tabela 3. Associação entre inatividade física e indicadores socioeconômicos conforme análise multivariável, João Pessoa - PB, 2005.

\begin{tabular}{|c|c|c|}
\hline \multirow{2}{*}{ Variável } & Rapazes & Moças \\
\hline & RP* (IC95\%) & RP* (IC95\%) \\
\hline \multicolumn{3}{|l|}{ Trabalho } \\
\hline Sim & 1 & 1 \\
\hline Não & $2,18(1,51-3,14)$ & $1,47(1,12-1,95)$ \\
\hline \multicolumn{3}{|c|}{ Tipo de escola } \\
\hline Pública & 1 & 1 \\
\hline Privada & $1,18(0,95-1,46)$ & $0,97(0,86-1,09)$ \\
\hline \multicolumn{3}{|c|}{ Classe econômica } \\
\hline$A, B$ & $0,94(0,72-1,23)$ & $1,01(0,81-1,16)$ \\
\hline C & $0,84(0,68-1,03)$ & $0,98(0,87-1,11)$ \\
\hline$D, E$ & 1 & 1 \\
\hline \multicolumn{3}{|c|}{ Escolaridade dos pais ${ }^{\dagger}$} \\
\hline$\leq 4$ anos & 1 & 1 \\
\hline 5-8 anos & $0,85(0,66-1,10)$ & $1,03(0,88-1,21)$ \\
\hline 9-11 anos & $0,92(0,71-1,19)$ & $1,18(1,02-1,38)$ \\
\hline$\geq 12$ anos & $0,93(0,69-1,24)$ & $1,23(1,03-1,46)$ \\
\hline
\end{tabular}

*Ajustado para idade e demais variáveis do modelo.

${ }^{\dagger}$ Chefe da família

\section{DISCUSSÃO}

O presente estudo demonstrou uma prevalência elevada $(55,9 \%)$ de inatividade física (demanda energética $<37 \mathrm{kcal} / \mathrm{kg} / \mathrm{dia}$ ) nos adolescentes escolares do ensino médio do município de João Pessoa - PB, assemelhando-se à que tem sido descrita por outros estudos com adolescentes Europeus ${ }^{(1,34)}$ e da região Sul do Brasi|(5,7), que utilizaram o mesmo critério de classificação do nível de atividade física.

Mesmo com diferenças em relação aos métodos e técnicas de medida da atividade física, e nos critérios e conceitos de inatividade física e/ou sedentarismo, outros estudos com adolescentes Europeus (60,9\% de sedentarismo: $<10 \%$ das atividades físicas no lazer, $\geq 4 \mathrm{MET})^{(2)}$, norte-americanos (64,2\% de insuficientemente ativos: $<60 \mathrm{~min} / \mathrm{dia}$, $<5$ vezes $/ \mathrm{sem})^{(4)}$, canadenses $(58,9 \%$ de inatividade física: $<3 \mathrm{kcal} / \mathrm{kg} /$ dia) $)^{(3)}$, e brasileiros da cidade de Porto Alegre - RS (39\% de sedentarismo: $<20 \mathrm{~min} / \mathrm{dia}$, $<3$ vezes $/ \mathrm{sem})^{(6)}$, também demonstraram prevalência elevada de adolescentes insuficientemente ativos.

Conforme vem sendo consistentemente descrito em diferentes estudos internacionais ${ }^{(1,3,4,35,36)}$ e nacionais ${ }^{(5-7)}$, a prevalência de inatividade física foi mais elevada nas moças $(64,2 \%)$ do que nos rapazes (45,5\%). Em estudo de revisão, Biddle et al.(21) verificaram que em vinte e dois, dos vinte e quatro estudos analisados ( $92 \%$ da amostra), as moças foram fisicamente menos ativas do que os rapazes, mostrando-se independente do instrumento de medida do conceito de inatividade física e/ou sedentarismo.

Essas diferenças se devem, entre outros fatores, ao maior envolvimento dos rapazes em atividades físicas mais intensas e, em menor escala, a maior duração e freqüência de prática de atividade física ${ }^{(35,36)}$. Torna-se necessário investigar os fatores que levam ao menor envolvimento das moças com atividade física de forma regular.

Como descrito previamente em outras investigações com adolescentes (14-18 anos) $)^{(1,5,7)}$, no presente estudo não se verificou tendência 
de redução nos níveis de atividade física com o passar da idade, nos adolescentes de ambos os sexos. Os estudos que têm descrito redução nos níveis de atividade física com o passar da idade foram desenvolvidos com adolescentes com faixa etária diferente e não mediram as atividades físicas em diferentes contextos ${ }^{(35,36)}$.

Tem-se observado que no período de transição da infância para adolescência ocorrem reduções expressivas no nível de atividade física da população jovem, sendo seguido de uma ligeira estabilização até o final da adolescência ${ }^{(3,35,36)}$. Nesse sentido, na população jovem, mudanças nos níveis de atividade física com passar da idade são dependentes da idade e do contexto da atividade física (lazer, locomoção, trabalho e vida diária) que está sendo analisada.

A condição socioeconômica, freqüentemente determinada a partir da renda familiar, grau de escolaridade, ocupação funcional, classe econômica, bens de consumo, ou combinação desses indicadores, está entre as principais variáveis em diversas teorias e modelos aplicados à atividade física. Esses indicadores favorecem a proximidade de alguns fatores que podem influenciar os hábitos de atividade física na população jovem ${ }^{(27,37)}$.

A análise da relação entre o nível de atividade física e a condição socioeconômica, mediante análise multivariável, evidenciou que adolescentes que não trabalhavam (rapazes: $\mathrm{RP}=2,18$ e moças: $\mathrm{RP}=1,47$ ) e cujos pais apresentam elevado grau de escolaridade (nas moças), estão mais expostos à inatividade física $(R P=1,23 ; I C=1,03-1,46)$, tendo como referência os seus pares que não trabalhavam e as moças que são filhas de pais com baixo grau de escolaridade ( $\leq 4$ anos). 0 tipo de escola (nos rapazes) e a classe econômica (nas moças), que na análise bruta haviam se associado estática e positivamente com a inatividade física, na análise ajustada perdeu significância estatística.

Não trabalhar durante a adolescência e ter pais com grau elevado de escolaridade são condições freqüentemente observadas em famílias de condições socioeconômicas mais privilegiadas. Nesse sentido, adolescentes mais ricos se mostraram mais expostos à inatividade física em comparação aos seus pares mais pobres, sobretudo as moças.

Corroborando os resultados do presente estudo, outras investigações que levaram em consideração as atividades físicas praticadas em diferentes contextos, também encontraram relação inversa entre o nível de atividade física e indicadores de condição socioeconômica(5,23,29).

Adolescentes que pertencem aos estratos socioeconômicos mais pobres referem maior utilização de transporte ativo (bicicleta e caminhada) no seu deslocamento diário (escola e trabalho) ${ }^{(23,29)}$, e as moças referem maior envolvimento em atividades do lar: cuidar da casa e dos irmãos, até mesmo trabalhar como babá e empregada doméstica para ajudar na renda familiar. Em países e regiões economicamente mais pobres, essas atividades apresentam uma contribuição ainda mais expressiva para o nível de atividade física dos adolescentes mais pobres.

Entretanto, outras investigações têm descrito menor nível de atividade física nos adolescentes que pertencem aos estratos socioeconômicas mais baixos ${ }^{(6,25,26,28)}$. É importante destacar que nessas investigações, a medida da atividade física se restringiu às atividades físicas realizadas no lazer, nas quais os adolescentes pobres apresentam menor envolvimento(6,28,38). Isso se deve às dificuldades na acessibilidade aos locais de prática, à baixa qualidade dos equipamentos disponíveis, à falta de recursos financeiros, e à insegurança ${ }^{(27,39)}$. A falta de tempo devido à responsabilidade com atividades profissionais e do lar, também são fatores que limitam o envolvimento com atividades físicas no lazer nesse subgrupo populacional(27,29).

Mesmo que os adolescentes que pertencem às famílias de menor condição socioeconômica tenham apresentado menor prevalência de inatividade física, do que os seus pares mais ricos, deve-se considerar que pertencer às classes socioeconômicas menos privilegiadas condiciona a uma maior participação em algumas atividades físicas que não são realizadas como forma de lazer, mas sim por "necessidade". Por outro lado, a baixa condição socioeconômica dos pais parece criar barreiras que dificultam a participação dos adolescentes em atividades físicas no lazer.

Embora não tenha sido analisada a relação entre a condição socioeconômica e a participação em atividades físicas em cada contexto de forma isolada (lazer, trabalho, locomoção, lar), é provável que haja diferença quanto à contribuição das atividades físicas realizadas em cada contexto, para o nível de atividade física, conforme a condição socioeconômica dos adolescentes: maior participação em atividades de lazer por parte dos adolescentes mais ricos e maior participação em atividades como meio de locomoção, trabalho e do lar entre os adolescentes mais pobres.

As discrepâncias entre os diferentes estudos acerca da relação condição socioeconômica versus nível de atividade física em adolescentes, em parte, podem ser explicadas pelos diferentes indicadores de condição socioeconômica, os métodos de medida da atividade física, definições de sedentarismo e inatividade física e, principalmente, pelo tipo de atividade física mensurada, utilizados nos diferentes estudos.

A utilização da quantidade de bens de consumo como um dos principais componentes para determinar a classe social dos adolescentes, pode não discriminar bem a classe econômica dos adolescentes. Tendo em vista que na última década houve uma grande disseminação desses produtos entre as classes mais pobres, em função do ligeiro aumento no poder de compra do salário mínimo, associado às facilidades para a aquisição desses bens. Além disso, as informações sobre o número de bens de consumo na residência e o grau de escolaridade dos pais, foram fornecidas pelos filhos, que podem gerar um viés na informação, em função da dificuldade de recordar essas informações.

Embora o nível de atividade física tenha sido determinado a partir de um diário de atividade física, que é uma medida subjetiva de atividade física, tem-se observado que os diários de atividade física apresentam níveis elevados de reprodutibilidade ${ }^{(30)}$ e se mostram instrumentos validados para medir o nível de atividade física em adolescentes ${ }^{(40)}$.

A prevalência de inatividade física no presente estudo foi elevada e afeta predominantemente às moças e indistintamente os adolescentes de todas as idades estudadas (14-18 anos), assemelhando-se ao que tem sido descrito na literatura.

Por se tratar de um estudo de caráter transversal, não é possível estabelecer uma relação de causa-efeito entre a posição socioeconômica e o nível de atividade física. Contudo, quando se determina o nível de atividade física a partir de uma medida que engloba as atividades físicas realizadas em diferentes contextos, a prevalência de inatividade se mostrou mais elevada nos adolescentes mais ricos: nos rapazes que não trabalham e nas moças que não trabalham e cujos pais apresentam maior grau de escolaridade.

Para tanto, torna-se necessário identificar os fatores que levam a diferenças no tipo e grau de envolvimento nas atividades físicas em vários contextos, entre os adolescentes de diferentes condições socioeconômicas. Essas informações podem ajudar a direcionar os objetivos e as ações dos programas de promoção da atividade física, com intuito de modificar os fatores que interferem nas escolhas dos adolescentes em relação às atividades físicas e favorecer maior envolvimento com a prática regular da atividade física, em todos os estratos socioeconômicos da população jovem.

Todos os autores declararam não haver qualquer potencial conflito de interesses referente a este artigo. 


\section{REFERÊNCIAS BIBLIOGRÁFICAS}

1. Cantera-Garde MA, Devís-Devís J. Physical activity levels of secondary school Spanish adolescent. Eur J Physical Education 2000; 5: 28-44.

2. Varo JJ, Martinez-González, Irala-Estévez J, Kearney J, Gibney M, Martinez JA. Distribution and determinants of sedentary lifestyles in the European Union. Int J Epidemiology 2003; 32: 138-46.

3. Koezuka N, Koo M, Allison KR, Adlaf EM, Qwyer JJM, Faulkner G, et al. The relationship between sedentary activities and physical inactivity among adolescents: results from the Canadian community health survey. J Adolesc Health 2006; 39: 515-22.

4. Centers for Disease Control and Prevention. Youth Risk Behavior Surveillance - United States, 2005. Surveillance Summaries, 2006. MMWR 2006; 55(No.SS-5).

5. Guedes DP, Guedes JERP, Barbosa DS, Oliveira JA. Níveis de prática de atividade física habitual em adolescentes. Rev Bras Med Esporte 2001; 7: 187-200.

6. Oehlschlaeger MHK, Pinheiro RT, Horta B, Gelatti C, Sant'Ana P. Prevalência e fatores de associados ao sedentarismo em adolescentes de área urbana. Rev Saúde Pública 2004; 38: 157-63.

7. Farias Júnior JC. Prevalência e fatores de influência para inatividade física em adolescentes. $\mathrm{R}$ bras $\mathrm{C}$ e Mov 2006; 14: 57-64

8. Adams J. Trends in physical activity and inactivity amongst US 14-18 year olds by gender, school grade and race, 1993-2003: evidence from the youth risk behavior survey. BMC public [serial online] 2006 [cited 200729 March]; 4:4 Available from: URL:http://www.biomedcentral.com/1471-2458/6/57.

9. Nelson MC, Neumark-Stzainer D, Hannan PJ, Sirad JR, Story M. Longitudinal and secular trends in physical activity and sedentary behavior during adolescence. Pediatrics 2006; 118: Available from: www.pediatrics.org/cgi/doi/10.1542/peds.

10. Duarte JA, Ribeiro JC, Oliveira J, Mota J. Relação entre níveis de atividade física e valores de colesterolemia em crianças e adolescentes. Rev Bras Saúde Mater Infant 2004; 4: 185-92.

11. Andersen LB, Harro M, Sardinha LB, Froberg K, Ekelund U, Brage S, et al. Physical activity and clustered cardiovascular risk in children: a cross-sectional study (the European youth heart study. Lancet 2006; 368: 299-304.

12. Guedes DP, Guedes JERP, Barbosa DS, Oliveira JA, Stanganelli LCR. Fatores de risco cardiovascular em adolescentes: indicadores biológicos e comportamentais. Arq Bras Cardiol 2006; 86: 439-50.

13. Pate RR, Health GW, Dowda M et al, Associations between physical activity and other health behaviors in a representative sample of US adolescents. Am J Public Health 1996; 86: 1577-81.

14. Gordon-Larsen P, Nelson MC, Popkin BM. Longitudinal physical activity and sedentary behavior trends Am J Prev Med 2004; 27: 277-83

15. Azevedo MR, Araújo CL, Silva CM, Hallal PC. Tracking of physical activity from adolescence to adulthood: a population-baseb study. Rev Saúde Pública 2007; 42: 69-75

16. Pate RR, Ward DS, Saunders RP, Felton G, Dishman RK, Dowda M. Promotion of physical activity among high-school girls: a randomized controlled trial. Am J Public Health 2005; 95: 1582-7.

17. Tsorbatzoudis $\mathrm{H}$. Evaluation of a school-based intervention programs to promote physical activity: an application of the theory of planned behavior. Percept Mot Skills 2005; 101: 787-802.

18. Rhodes RE, Macdonald HM, Mckay HA. Predicting physical activity intention and behavior among children in a longitudinal sample. Soc Sci Med 2006; 62: 3146-56.

19. Stewart-Brown S. What is the evidence on school health promotion in improving health or preventing disease and, specifically, what is the effectiveness of the health promoting schools approach? Copenhagen, WHO Regional Office for Europe. Health Evidence Network Report, 2006.

20. Sallis JF, Prochaska JJ, Taylor WC. A review of correlates of physical activity of children and adolescents. Med Sci Sports Exerc 2000; 32: 963-75.
21. Biddle SJH, Whitehead SH, O'Donovan TM, Nevill ME. Correlates of participation in physical activity for adolescent girls: a systematic review of recent literature. J Physical Activity and Health 2005; 2: 423-34.

22. Gustafson SL, Rhodes RE. Parental correlates of physical activity in children and early adolescents. Sport Medicine 2006; 36: 79-97.

23. Hallal PC, Bertoldi AD, Gonçalves H, Victora CG. Prevalência de sedentarismo e fatores associados em adolescentes de 10-12 anos de idade. Cad Saúde Pública 2006; 22: 1277-87.

24. Gomes VB, Siqueira KS, Sichieri R. Atividade física em uma amostra probabilística da população do município do Rio de Janeiro. Cad Saúde Pública 2000; 17: 969-76.

25. Trost ST, Pate RR, Ward DS, Saundrs R, Riner W. Correlates of objectively measured physical activity in preadolescent youth. Am J Prev Med 1999; 17: 120-6.

26. Lasheras L, Aznar S, Merino B, López EG. Factors associated with physical activity among Spanish youth though the national health survey. Prev Med 2001; 32: 455-464.

27. Humbert ML, Chad KE, Spink KS, Muhajarine N, Anderson KD, Bruner MW, et al. Factors that influence physica activity participation among high-and low-SES youth. Qual Health Res 2006; 16: 467-83.

28. La Torre G, Massala D, De Vito E, Langiano E, Capelli G, Ricciardi W. Extra-curricular physical activity and socioeconomic status in Italian adolescents. BMC public health [serial online] 2006 [cited 2007 29 March]; 4:4 Available from: URL:http://www.biomedcentral.com/1471-2458/6/57.

29. Shi Z, Lein N, Kumar BN, Holmboe-Ottesen G. Physical activity and associated socio-demographic factors among school adolescents in Jiangsu Province, China. Prev Med 2006; 43: 218-1.

30. Farias Júnior JC, Pires MC, Lopes AS. Reprodutibilidade de um questionário para o levantamento de informações sobre comportamentos relacionados à saúde em adolescentes. R Bras Ci e Mov 2002; 10: 43-8.

31. ABEP (Associação Brasileira de Empresas de Pesquisa). Critério de Classificação Econômica Brasil. [www. abep.org.br]. Disponível em: www.abep.org.br/mural/abep/cceb.htm. Acessado em 26/11/2005.

32. Ainsworth BE, Haskell WL, Whitt MC, Irwin ML, Swartz AM, Strath SJ, et al. Compendium of physical ac tivities: an update of activity codes and MET intensities. Med Sci Sports Exerc 2000; 32: S498-S516.

33. Cale L. Monitoring physical activity in children. [Phd Thesis]. Lougborough: Department of Physical Education, Sport, Science and Recreation Management, University of Technology; 1993.

34. Cale L, Almond L. The physical activity levels of english adolescent boys. Eur J Physical Education 1997; 2: 74-82.

35. Telama R, Yang X. Decline of physical activity from youth to young adulthood in Finland. Med SC Sports Exerc 2000; 32: 1617-22.

36. Van Mechelen W, Twisk JWR, Post GB, Snel J, Kemper HCG. Physical activity of young people: the Amsterdam longitudinal growth and health study. Med Sci Sports Exerc 2000; 32: 1610-6.

37. Tammelin T, Näyhä S, Laitinen J, Rintamäki H, Järvelin M. Physical activity and social status in adoles cence as predictors of physical inactivity in adulthood. Prev Med 2003; 37: 375-81.

38. Santos MP, Esculcas C, Mota J. The relationship between socioeconomic status and adolescents organized and nonorganized physical activities. Pediatric Exercise Science 2004; 16: 210-8.

39. Romero AJ. Low-income neighborhood barriers and resources for adolescents' physical activity. J Adolesc Health 2005; 36: 253-9.

40. Wickel EE, Welk GJ, Eisenmann JC, Concurrent validation of the Bouchard diary with an accelerometrybased monitor. Med Sci sports Exerc 2006; 38: 373-9. 